\title{
Atractylenolide I inhibits antibiotic-induced dysbiosis of the intestinal microbiome
}

\author{
Penglin Liu ${ }^{1,2}$, Gang Zhao $^{2}$, Lize Zhang' ${ }^{2}$ Yuxia Gong ${ }^{3}$, Yunfei Gu ${ }^{1}$ \\ ${ }^{1}$ The First Clinical Medical College of Nanjing University of Chinese Medicine, Nanjing, China; ${ }^{2}$ Department of Proctology, Affiliated Hospital of \\ Qingdao University, Qingdao, China; ${ }^{3}$ Department of Anorectal Surgery, Affiliated Hospital of Nanjing University of Traditional Chinese Medicine, \\ Nanjing, China \\ Contributions: (I) Conception and design: All authors; (II) Administrative support: All authors; (III) Provision of study materials or patients: All \\ authors; (IV) Collection and assembly of data: All authors; (V) Data analysis and interpretation: All authors; (VI) Manuscript writing: All authors; (VII) \\ Final approval of manuscript: All authors. \\ Correspondence to: Yunfei Gu. The First Clinical Medical College of Nanjing University of Chinese Medicine, Nanjing 210023 , China. \\ Email: Dr.Guyunfei@hotmail.com.
}

Background: Atractylenolide I (AT-I) is an active component that is isolated from Rhizoma Atractylodis macrocephalae and it exerts anti-apoptotic, anti-oxidant, and anti-coagulant properties, and has been widely applied in the treatment of cardiovascular and cerebrovascular diseases in China. This study aimed to investigate the effects and possible mechanism of AT-I on intestinal dysbacteriosis in a mouse model.

Methods: Mice dysbacteriosis models were established and treated with AT-I, and the intestinal microbiome of the mice were compared. Using antibiotics-induced bacterial elimination in an intestinal dysbacteriosisassociated xenograft model, the gut microbiota-mediated anti-tumor mechanism was investigated.

Results: The intestinal microbiome was changed in the dysbacteriosis mice compared to the control mice, and AT-I could affect the intestinal microbiome of the dysbacteriosis mice. Manipulation of gut bacteria in the intestines of the dysbacteriosis-associated xenograft model further confirmed that the inhibition of tumor progression by AT-I was mediated by the gut microbiota, and that the underlying mechanism involves downregulation of TLR4/MyD88/NF- $\kappa \mathrm{B}$ signaling. AT-I repressed the phosphorylation of $\mathrm{p} 65-\mathrm{NF}-\kappa \mathrm{B}$ as well as the downstream cytokines, IL-6 and IL-1 $\beta$, in dysbacteriosis mice.

Conclusions: AT-I may inhibit dysbacteriosis by affecting the intestinal microbiome via the regulation of TLR4/MyD88/NF- $\mathrm{B}$ signaling. The present study provides a basis for the application of AT-I as an alternative medication for treating gastrointestinal disorders.

Keywords: Atractylenolide I (AT-I); intestinal dysbacteriosis; NF-кB

Submitted Aug 10, 2021. Accepted for publication Oct 21, 2021.

doi: $10.21037 / \mathrm{atm}-21-4656$

View this article at: https://dx.doi.org/10.21037/atm-21-4656

\section{Introduction}

Previous studies have proposed a potential role of the gut microbiome in gastrointestinal disorders (1-3). Intestinal dysbacteriosis, which refers to abnormal changes of the intestinal microbiome, may prompt an aberrant immune response in colorectal tissue, which further induces chronic inflammation. This causes the epithelial-mesenchymal transition (EMT) of epithelial cells, and ultimately leads to a chronic malignant condition and even the development of tumors $(4,5)$. Therefore, alleviating intestinal dysbacteriosis may be an alternative approach for the treatment of different types of gastrointestinal disorders.

Atractylenolide I (AT-I) is an active component that is isolated from Rhizoma Atractylodis macrocephalae (a traditional medication) (6-9). It exerts anti-apoptotic, anti-oxidant, and anti-coagulant properties, and has been widely applied in the treatment of cardiovascular 
and cerebrovascular diseases in China (10-12). In recent years, the therapeutic effects of AT-I in the treatment of gastrointestinal disorders have been discussed in numerous studies $(7,9,13,14)$.

A large number of microorganisms are colonized in the human intestine, which plays an indispensable role in intestinal function. Furthermore, the intestinal flora plays a key role in inhibiting the production of intestinal tumor cells. When the normal flora is in an abnormal state and the balance with the host is broken, diseases such as inflammatory bowel disease will occur (15). In recent years, many scholars have shown that AT-I can regulate the intestinal microecology and improve intestinal diseases to a certain extent, with the main material basis being AT-I polysaccharide. In a mouse model of intestinal flora imbalance created by lincomycin, Shu et al. found that AT-I decoction can reduce the $\mathrm{pH}$ of the mouse intestinal microenvironment, increase the adhesion of intestinal mucosal epithelial cells to bifidobacteria, support normal intestinal flora, and inhibit the proliferation of Escherichia coli and Enterococcus faecalis (16). In addition, studies have shown that AT-I decoction also has obvious inhibitory influences on Escherichia coli, Staphylococcus aureus, and methicillin-resistant Staphylococcus aureus, among other pathogenic bacteria, in a dose-response relationship, and has a certain effect on maintaining the steady state of intestinal bacteria (17). Wang et al. (18) confirmed in the anaerobic culture of AT-I polysaccharide on intestinal flora that AT-I polysaccharide can promote the digestion of reducing sugar by intestinal bacteria, improve symptoms of watery stool created by senna, enhance the similarity coefficient of the Enterobacterial Repetitive Intergenic Consensus-polymerase chain reaction (ERIC-PCR) fingerprint of intestinal flora DNA, regulate the structure of intestinal flora and restore its homeostasis, and can be implemented as an adjuvant to regulate intestinal flora.

Due to the large distribution of the intestinal flora and the imbalance of the intestinal flora in the colon in the human intestine, it has a strong inducing effect on the occurrence of colorectal cancer. Imbalance of the intestinal flora leads to impaired protection of the intestinal mucosa. Various metabolites and bacteria will translocate, bacterial endotoxins are released, and the intestinal mucosa is destroyed (19). Cells have lost their original protective barrier and are more susceptible to carcinogens. The imbalance of intestinal flora homeostasis causes changes in bacterial metabolism, changes in metabolites, and induces the occurrence of colorectal cancer (20).

Under normal circumstances, enzymes produced by the intestinal flora regulate tumor growth by activating or inhibiting carcinogens. Under the condition of intestinal flora imbalance, allergic bowel disease or inflammatory bowel disease will occur in the intestine due to the imbalance of corresponding enzymes, leading to the production of carcinogens, and then colon cancer. Changes in the number of certain beneficial or harmful bacteria can disrupt the balance of intestinal flora, leading to the possibility of colon cancer. By analyzing the difference in bacterial composition in the stool of healthy people and colorectal cancer patients, it was found that a variety of beneficial bacteria and bacterial diversity in the stool of colorectal cancer patients decreased, and the proportion of harmful bacteria increased significantly $(21,22)$.

Atractylodes macrocephala can effectively inhibit the growth and proliferation of tumor cells. There have been some experiments have confirmed that its lactones are the basis for achieving anti-tumor effects (23). Related studies have found that Atractylodes I has good anti-cancer activity and can significantly inhibit the vitality of a variety of tumor cells, including MCF7, HepG, Dul45, A549, A875, P-388 and HL-60 (24-26). Atractylodes lactone I can also induce apoptosis of leukemia cells and produce cytotoxic effects (27). In vivo experimental study of antibiotic associated diarrhea in rats found that after Shenlingbaizhu powder, the main active ingredient is Atractylodes macrocephala, after treatment, the $\mathrm{P}$ substance and endotoxin in the mice were reduced, and the probiotic content increased. It is known that Atractylodes macrocephala has a certain regulatory effect on the pathological phenomenon of intestinal flora imbalance caused by antibiotics (28).

However, it remains unclear whether AT-I could affect intestinal dysbacteriosis and therefore exert therapeutic effects. In this study, we investigated the influences and underlying mechanism of AT-I on intestinal dysbacteriosis mice. Our findings may provide a novel mechanism and alternative therapeutic strategy for patients with gastrointestinal disorders. We present the following article in accordance with the ARRIVE reporting checklist (available at https://dx.doi.org/10.21037/atm-21-4656).

\section{Methods}

\section{Intestinal dysbiosis mouse model}

Mice were purchased from Beijing Vital River Laboratory Animal Technology Co., Ltd. An intestinal dysbiosis mouse model was established as previously described (5). Mice were 
orally treated with ampicillin, vancomycin, neomycin, and metronidazole to induce intestinal dysbiosis. Experiments were performed under a project license (No. 2020-039) granted by ethics board of Affiliated Hospital of Nanjing University of Traditional Chinese Medicine, in compliance with $\mathrm{NIH}$ guidelines for the care and use of animals.

\section{Cell lines and culture}

HCT116 and LoVo cells were resuscitated using the conventional cell culture method, and were then diluted to a certain density with Dulbecco's modified Eagle's medium (DMEM) high glucose medium containing $10 \%$ fetal bovine serum and cultured in a constant temperature incubator (Suzhou Jiemei Electronics Co., Ltd., China) of $37{ }^{\circ} \mathrm{C}$ and $5 \% \mathrm{CO}_{2}$. Cells in the logarithmic growth phase were collected for follow-up experiments.

\section{Cell viability assay}

A Cell Counting Kit-8 (CCK-8; Dojindo, Japan) assay was performed to detect the effect of AT-I on the viability of HCT116 and LoVo cells. Briefly, HCT116 and LoVo cells were respectively seeded into 96-well plates at an initial density of $5 \times 10^{3}$ cells/well. After 24 hours of drug intervention, $20 \mu \mathrm{L}$ of CCK- 8 reagent was added to each well, and then incubated in an incubator for 2 hours. The culture medium was aspirated in the well, and the absorbance [optical density (OD) value] of each well was measured at $450 \mathrm{~nm}$ with a microplate reader (Shanghai peiou Analytical Instrument Co., Ltd., China) to calculate cell viability. The experiment was repeated three times.

\section{Western blotting}

Briefly, a part of the tissue was taken and an appropriate amount of liquid nitrogen was added. The tissue was then ground using a mortar and lysis buffer was added. It was then homogenized it in the tissue homogenizer (Guansen biotechnology Shanghai Co., Ltd., China); the tissue was crushed as much as possible, and stood on ice for 15-30 min for cracking. The homogenate was then placed into a centrifuge tube at 3,000 r/min for centrifugation for $10 \mathrm{~min}$. Subsequently, the supernatant was transferred to a new precooled centrifuge tube, the protein was treated, and the sample was loaded.

The total protein was separated by sodium dodecyl sulfate-polyacrylamide gel electrophoresis (SDS-PAGE) and then transferred to a polyvinylidene fluoride (PVDF) membrane using the wet transfer method. The primary antibodies (Bcl-2, Bcl-xL, GAPDH, TLR4, MyD88, p-ikba, IK Ba, p-p65, p65) were then diluted with $5 \%$ skimmed milk powder (dilution ratio: $1: 1,000$ ), added to a PVDF membrane, incubated overnight at $4{ }^{\circ} \mathrm{C}$, and then incubated with secondary antibody at room temperature for 2 hours. Image J software (National Institutes of Health, Bethesda, USA) was used for gray value analysis.

\section{Enzyme-linked immunosorbent assay (ELISA) assay}

The expression levels of interleukin (IL)-6 and IL-1 $\beta$ were examined using ELISA. The ELISA was conducted with kits purchased from Beyotime (Shanghai, China), and all procedures were performed strictly according to the manufacturer's protocols.

\section{Histopathological analysis}

The mice were euthanized by $\mathrm{CO}_{2}$ asphyxiation and punctured segments of tumor tissue were collected. The tissue was embedded in paraffin and continuously cut into $5 \mu \mathrm{M}$-thick slices. The slides of each disc were stained with hematoxylin and eosin (H\&E). Two gastrointestinal pathologists from Shuguang Hospital examined the histopathological changes of the stained sections.

\section{TdT-mediated dUTP-biotin nick end labeling (TUNEL) assay}

TUNEL staining was applied to the prepared paraffin sections to observe apoptosis. The tissue was stained in strict accordance with the steps in the TUNEL kit manual, and was observed under the microscope $(\times 400)$ (Thermo Fisher Scientific, Waltham, Massachusetts, USA). Five visual fields were selected in the ischemic edge area of each pathological section, the number of TUNEL positive cells was counted using Image Pro Plus image analysis software (MEDIA CYBERNETICS, Maryland, USA), and the average value was taken as the number of positive cells of the sample. Percentage of apoptotic cells $(\%)=$ number of positive apoptotic cells/(number of positive apoptotic cells + number of negative apoptotic cells) $\times 100 \%$.

\section{Statistical analysis}

All experiments were carried out in triplicate. All data 
Table 1 Comparison of the abundances of different microorganisms in the intestinal microbiome of dysbacteriosis mice with or without AT-I treatment

\begin{tabular}{lccc}
\hline Species & Dysbacteriosis & AT-I low & AT-I high \\
\hline Lactobacillus & $12.74 \pm 1.56$ & $18.51 \pm 2.07$ & $27.23 \pm 3.18^{\star \star}$ \\
Bacteroides & $19.32 \pm 1.88$ & $24.36 \pm 1.59^{\star}$ & $28.61 \pm 2.94^{\star}$ \\
Escherichia & $2.51 \pm 0.29$ & $1.61 \pm 0.14$ & $0.34 \pm 0.02^{\star \star}$ \\
Candidatus & $8.39 \pm 0.84$ & $5.37 \pm 0.55^{\star}$ & $3.39 \pm 0.68^{\star \star}$ \\
\hline
\end{tabular}

*, $\mathrm{P}<0.05$ vs. dysbacteriosis; ${ }^{\star *}, \mathrm{P}<0.01$ vs. dysbacteriosis. AT-I, atractylenolide I.

were investigated using SPSS 19.0 software (IBM, New York, USA). The values were presented as mean \pm standard deviation (SD). Statistical significance was investigated using unpaired Student's $t$-test. $\mathrm{P}<0.05$ was generally considered to be statistically significant.

\section{Results}

\section{AT-I changes the intestinal microbiome}

To determine whether AT-I can inhibit the growth of dysbacteriosis by regulating the intestinal microbiome, we treated the dysbacteriosis mice with AT-I (as the model group). The microbiome in the intestines of the AT-I and control groups were compared. We found that AT-I increased the abundance of Lactobacillus and Bacteroides, and decreased the abundance of Escherichia and Candidatus in a dose-dependent manner (Table 1).

\section{Effect of AT-I on serum lipopolysaccharide (LPS) and IL-1及 levels in antibiotics-treated mice}

In order to explore the effect of AT-I on bacterial flora-related signal molecules, the LPS levels in serum, feces, and colon homogenate were determined. As shown in Figure 1A-1C, the serum LPS level of the antibiotics-treated group was higher than that of the control group $(\mathrm{P}<0.001)$, and the intervention of AT-I and probiotics significantly restrained the release of LPS $(\mathrm{P}<0.01$ and $\mathrm{P}<0.01$, respectively). The same outcomes were obtained for LPS levels in the stool and colon homogenate, indicating that after intervention with antibiotics, the overall level of LPS levels in mice increased (which has a significant relationship with the intestinal flora), and AT-I and probiotics reversed this increase. The serum IL- $1 \beta$ concentration was measured at the same time, and the possible inflammatory reaction was observed. As shown in Figure $2 A$, the serum IL-1 $\beta$ levels of the antibiotics-treated mice was markedly higher than that of the control group $(\mathrm{P}<0.001)$, and treatment with AT-I restrained these levels $(\mathrm{P}<0.01)$.

\section{The effect of AT-I on colonic pathology and cell apoptosis}

To explore how AT-I reduced tumorigenesis in model-group mice, we evaluated the morphological and pathological influences of AT-I in the xenograft mice. As shown in Figure 2B, the colonic mucosa of the normal group was intact; the epithelial cells were arranged neatly without inflammatory cell infiltration. In the model group, the colonic mucosa was obviously missing; the lamina propria glands were damaged or disappeared, goblet cells were restrained, and there was inflammatory cell infiltration. In the AT-I and probiotic groups, the epithelial cell defect was not obvious; the basic morphology was similar to that of the normal group.

TUNEL staining outcomes revealed that tumor cell apoptosis was significantly diminished in the model group. Meanwhile, AT-I evidently eliminated this apoptotic effect and promoted tumor cell apoptosis (Figure $3 A, 3 B$ ). It is well known that $\mathrm{Bcl}-2$ and $\mathrm{Bcl}-\mathrm{xL}$ proteins are involved in inhibiting tumor cell apoptosis. Western blot outcomes showed that the expression levels of anti-apoptotic proteins $\mathrm{Bcl}-2$ and $\mathrm{Bcl}-\mathrm{xL}$ in the tumor tissues of the model group increased, and AT-I reversed this change (Figure 3C-3E). Therefore, we believe that AT-I inhibits tumor growth through pro-apoptotic influences.

\section{The anti-tumor mechanism of AT-I based on intestinal microbes is involved in the inbibition of the TLR4/

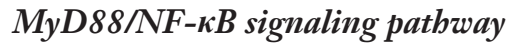

Since AT-I regulates the intestinal bacteria that produce LPS, and pro-inflammatory cytokines are closely related to the TLR4/MyD88/NF-kB pathway, we speculate that the anti-tumor effect of AT-I may be related to the TLR4 
A

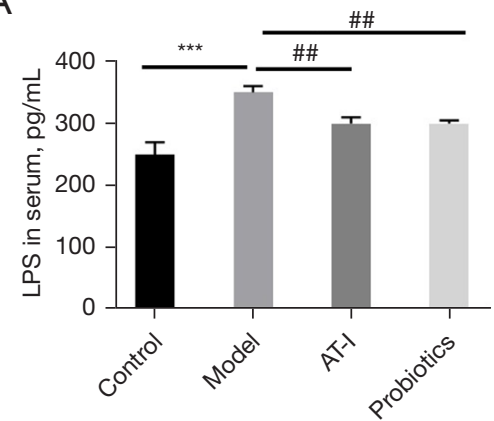

B

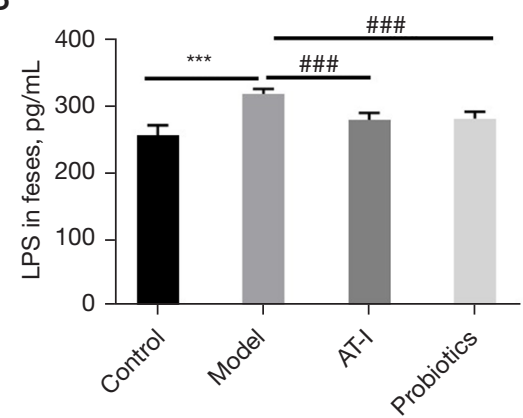

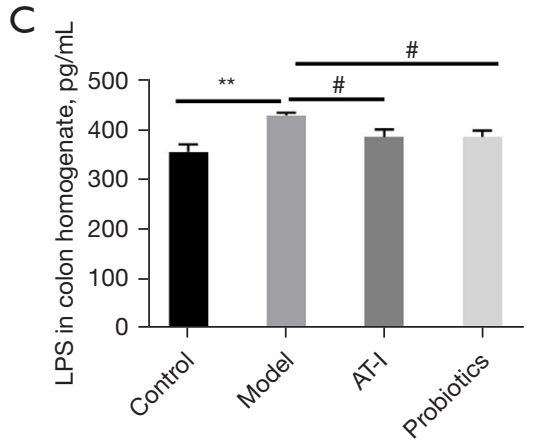

Figure 1 The effect of AT-I on antibiotic-treated mice gut microbiota and LPS levels. The effect of AT-I on (A) serum LPS levels, (B) fecal

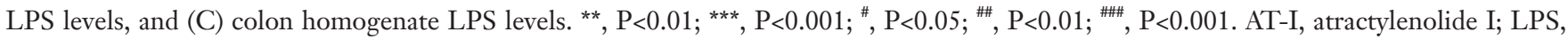
lipopolysaccharide.

A

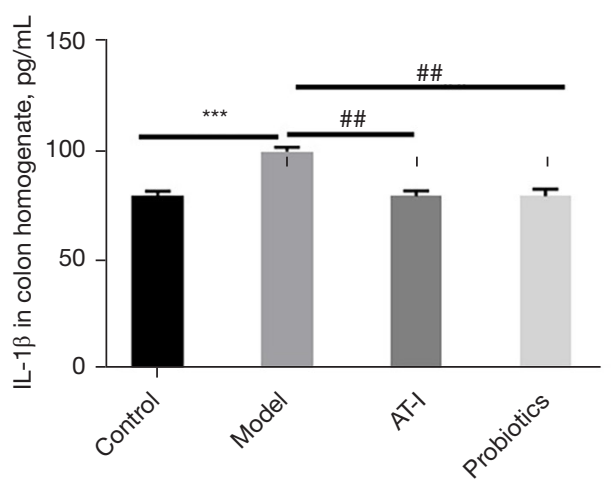

B

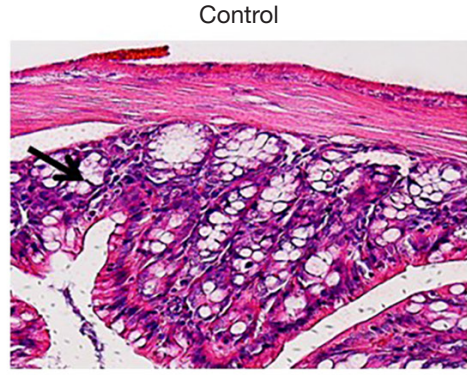

AT-I

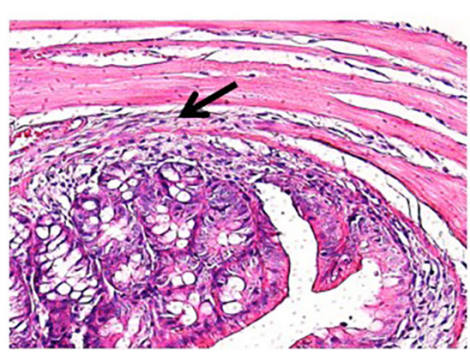

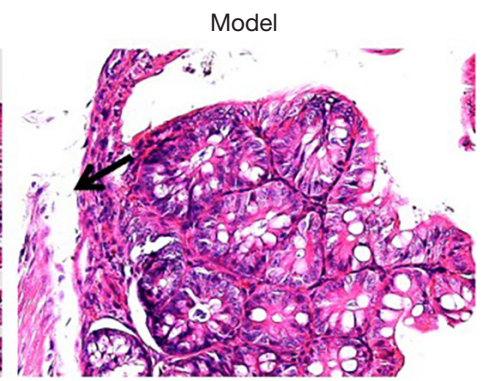

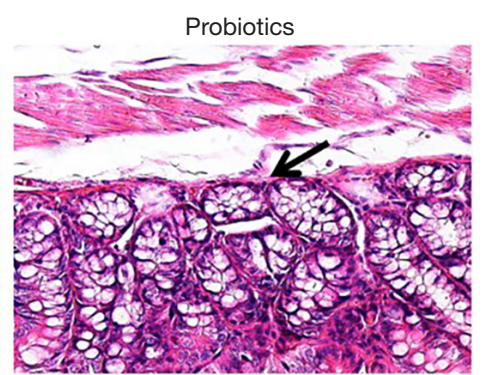

Figure 2 The effect of AT-I on IL-1 $\beta$ and the effect of AT-I on colonic pathology. (A) Serum IL-1 $\beta$ levels. (B) The effect of AT-I on colonic pathology. Colon H\&E staining, magnification $400 \times$. The arrows represent the structural integrity of the intestine. ${ }^{* * *}, \mathrm{P}<0.001 ;{ }^{*}, \mathrm{P}<0.01$. AT-I, atractylenolide I; IL, interleukin; H\&E, hematoxylin and eosin.

signaling pathway.

These results indicate that AT-I can inhibit the activation of the TLR4/MyD88/NF- $\mathrm{kB}$ signaling pathway. In order to further explore the anti-tumor mechanism of AT-I microbes, we tested the microbes-mediated tumor immune signaling pathway. As shown in Figure 4A-4E, TLR4, $\mathrm{MyD} 88$, and the ratio of $\mathrm{p}-\mathrm{IkB} / \mathrm{IkB}$ and $\mathrm{p}-\mathrm{p} 65 / \mathrm{p} 65$ were obviously restrained in the AT-I-H and ABX + AT-I-H groups. These results indicate that the potential anti-tumor mechanism of AT-I is related to the TLR4/MyD88/NF- $\mathrm{kB}$ signaling pathway and is mediated by intestinal microbes.

\section{Intestinal bacteria mediated the effect of AT-I on the tumor phenotype of xenotransplanted mice suffering from intestinal dysbiosis}

In order to explore whether AT-I affects cancer progression, we first tested the direct anti-tumor effect of AT-I. 

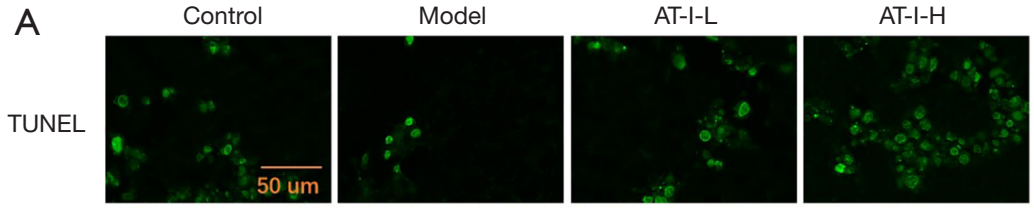

DAPI
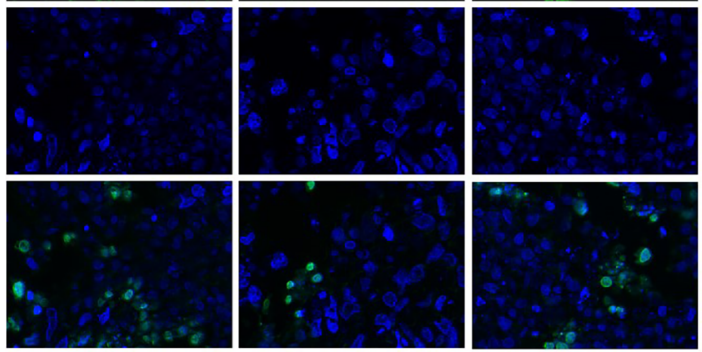

C

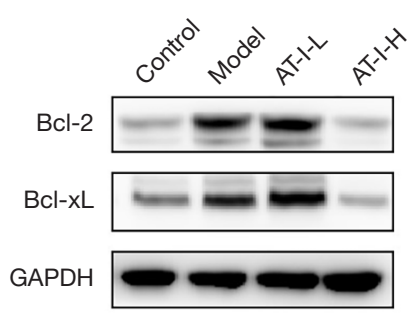

D

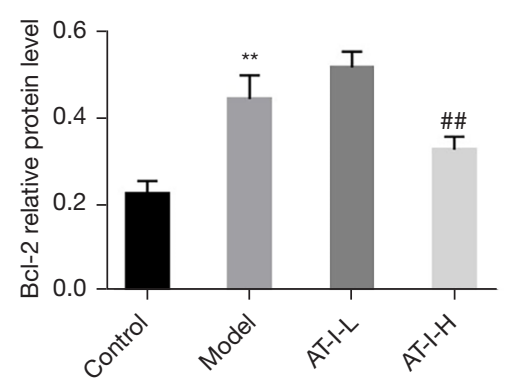

B

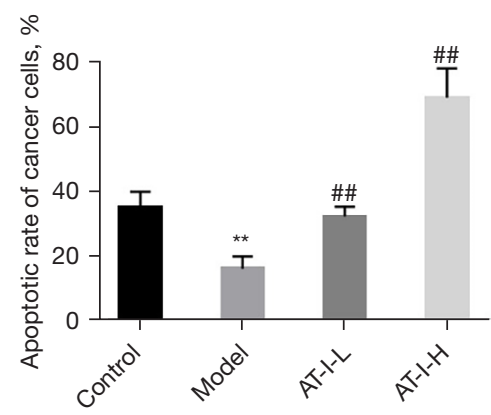

E

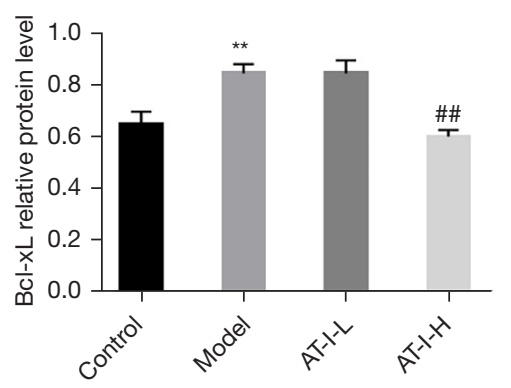

Figure 3 AT-I inhibits tumor cell apoptosis with imbalanced intestinal flora in xenotransplanted mice. (A,B) TUNEL staining to detect the apoptosis rate of cancer cells in tumor tissue. (C-E) Bcl-2 and Bcl-xL protein expression in each group. ${ }^{* *}, \mathrm{P}<0.01$; ${ }^{\# \#}, \mathrm{P}<0.01$. AT-I, atractylenolide I.

In vitro outcomes showed that AT-I had no effect on the cell viability of HCT 116 and LoVo cells (Figure 5A,5B). In vivo experiments of transplantation models demonstrated that AT-I has no effect on tumor growth, while 5-FU was found to significantly inhibit tumor growth (Figure 5C,5D). These results indicate that AT-I has no direct anti-tumor effect.

\section{AT-I is mediated by intestinal flora in xenotransplanted models}

Considering the role of intestinal flora in tumorigenesis and intestinal flora imbalance, we hypothesized that intestinal flora might mediate the efficacy of AT-I in the treatment of cancer with intestinal flora imbalance. For this reason, during the AT-I intervention, antibiotics depleted the residual flora of mice with intestinal disorders. Compared with the AT-I group, the tumor volume and weight of the antibiotic + AT-I-H group were statistically significant (Figure 6A,6B), suggesting that the consumption of intestinal bacteria weakened the anti-tumor effect of AT-I in mice with intestinal disorders. As shown in Figure $6 C, 6 \mathrm{D}, \mathrm{Bcl}-2$ in the AT-I-H and ABX + AT-I-H groups was obviously restrained compared to the model group. Also, there was no significant difference in Bcl-xL between the ABX + AT-I-H group and the model group. In summary, these results indicate that the anti-tumor effect of AT-I in xenotransplanted models (related to intestinal flora disorders) is mediated by intestinal flora.

\section{Discussion}

The role of the gut microbiome in different type of diseases has been discussed in many previous studies. At present, intestinal dysbacteriosis is considered an important factor during the development of diseases $(2,4,19-21)$. The therapeutic influences of AT-I have been discussed in numerous studies; however, reports on the effect of AT-I on the gut microbiome are rare. The mechanism of AT-I regulating gastrointestinal movement is complex. The 

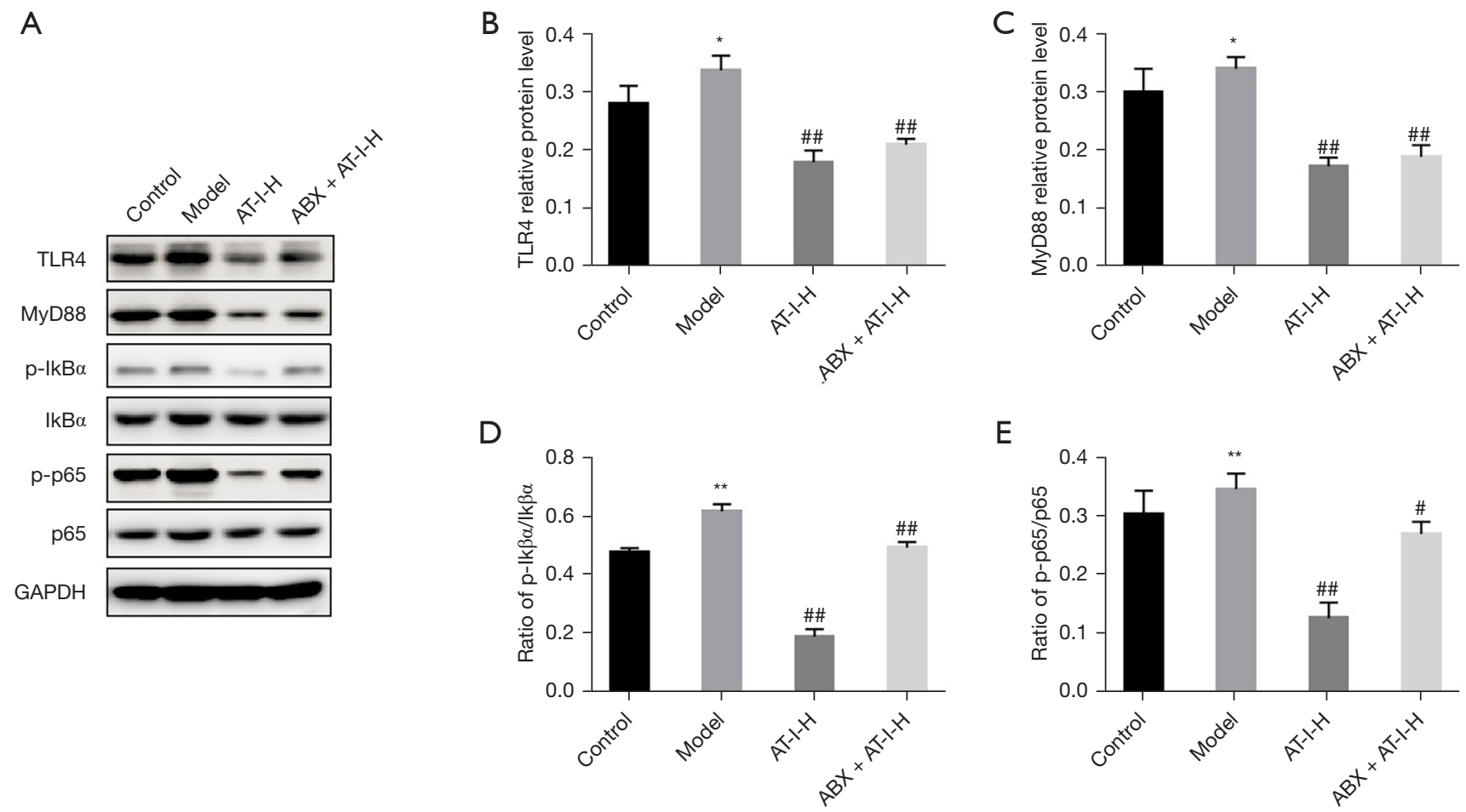

Figure 4 The anti-tumor mechanism of AT-I based on the intestinal microbiota participates in inhibiting the four groups of TLR4/MyD88/

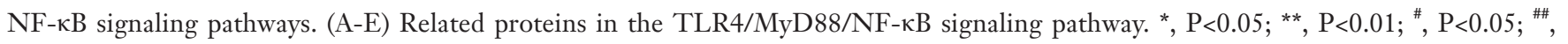
$\mathrm{P}<0.01$. AT-I, atractylenolide I.

current research shows that its mechanism is related to the intestinal nervous system, gastrointestinal hormones, and neurotransmitters of the gastrointestinal tract. For example, interstitial cells of Cajal (ICC) are the basic structural unit of the intestinal nervous system. These are independent special interstitial cells, which exist in the muscular layer and mucosa of the gastrointestinal tract in a reticular structure with nerve cells. Cajal stromal cells are the pacemaker cells of the gastrointestinal tract and can spontaneously produce slow waves. Therefore, ICCs play an important role in the regulation of gastrointestinal movement (22).

Studies have shown that the aqueous extract of AT-I can significantly increase the contents of motilin, vasoactive intestinal peptide, and somatostatin in plasma and jejunum of cirrhotic model rats, and markedly reduce the contents of cholecystokinin and improve their gastrointestinal function (23). Xu et al. conducted an in vitro experiment of AT-I polysaccharide co-incubated with intestinal bacteria, and showed that the metabolic rate of AT-I polysaccharide is obviously higher than that of artificial gastric and intestinal juices; that is, the metabolism of AT-I polysaccharide mainly occurs in the intestine (24). Pan et al. (25) investigated the growth-promoting effect of AT-I polysaccharide on probiotic bifidobacteria and Lactobacillus. Their results demonstrated that the addition of $1.0 \%$ AT-I polysaccharide had a good growth-promoting effect on L. acidophilus, and that the metabolism of AT-I polysaccharide and the growth of intestinal probiotics could interact and promote each other. In the present study, we first reported the effect AT-I on the intestinal microbiome. We found that AT-I increased the abundance of the beneficial microorganisms and decreased the levels of harmful microorganisms in dysbacteriosis mice, suggesting that AT-I could exert its therapeutic influences partially by affecting the intestinal microbiome.

Intestinal dysbacteriosis is known to induce chronic inflammatory conditions and ultimately lead to tumorigenesis. $\mathrm{NF}-\kappa \mathrm{B}$ is a complex of proteins that participate in the progression of immune responses $(26,27)$. In order to further explore the anti-tumor mechanism of AT-I microorganisms, we tested the tumor immune signaling pathway mediated by microorganisms. As shown in Figure 4A-4E, TLR4, 
A

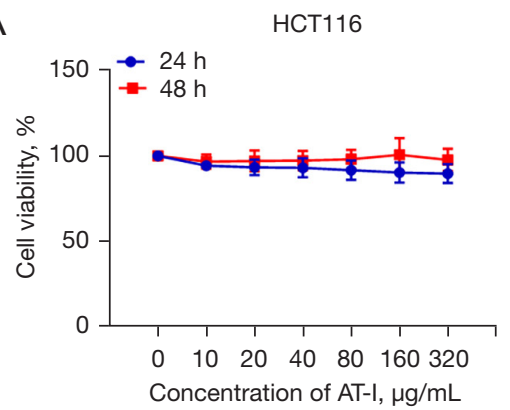

C

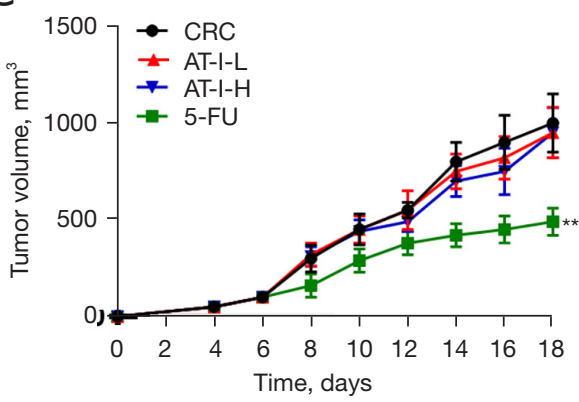

B

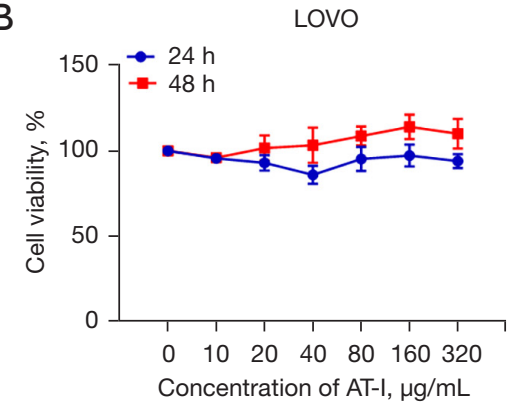

D

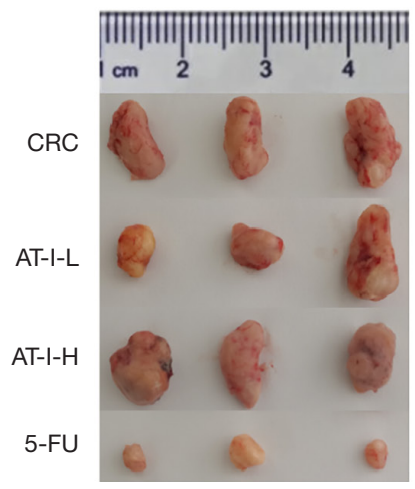

Figure 5 AT-I inhibits tumor growth in xenotransplanted mice with intestinal microflora disorders. (A,B) The cell viability of HCT116 and LoVo cells treated with AT-I was measured by CCK-8. Each treatment method was evaluated in triplicate. (C,D) Tumor size and tumor growth curve of xenograft mice ( $\mathrm{n}=3$ /group). ${ }^{* *}, \mathrm{P}<0.01$. AT-I, atractylenolide I; CCK-8, Cell Counting Kit- 8 .

A

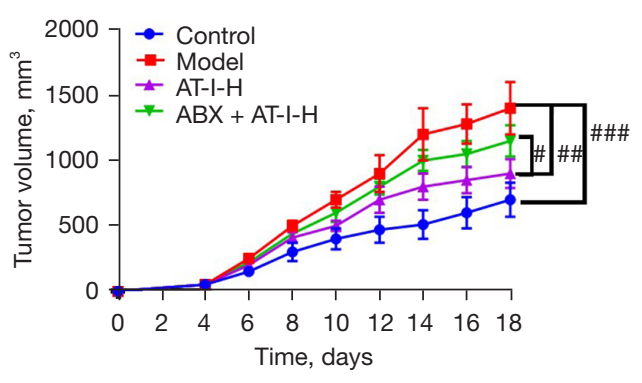

C

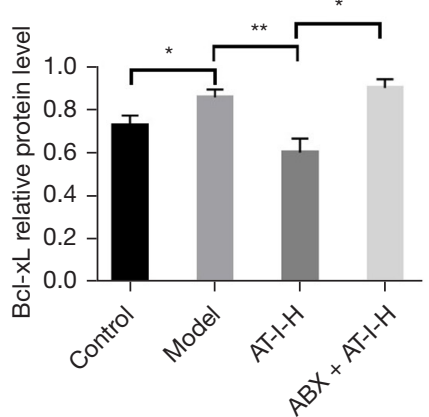

B

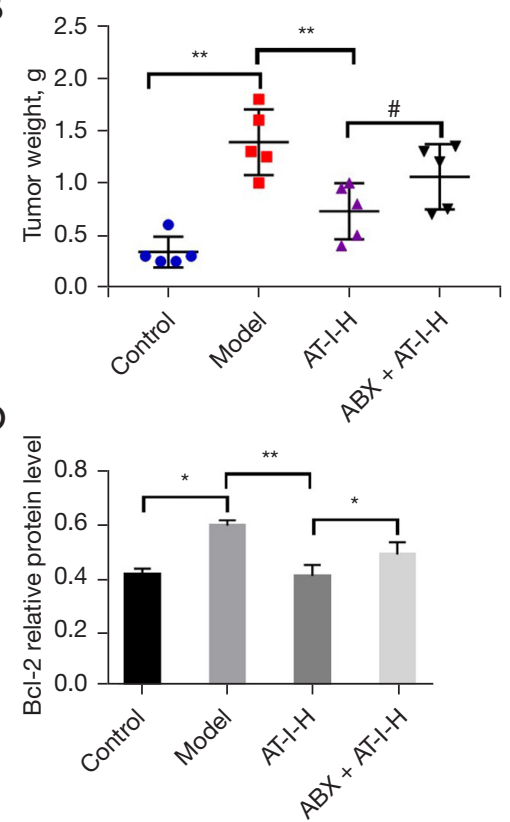

Figure 6 Intestinal bacteria mediated the effect of AT-I on the tumor phenotype of xenotransplanted mice suffering from intestinal dysbiosis. (A,B) Tumor growth and xenograft weight treatment. (C,D) Bcl-2 and Bcl-xL proteins in the four groups. ${ }^{*}, \mathrm{P}<0.05 ;{ }^{* *}, \mathrm{P}<0.01$; ${ }^{*}$, $\mathrm{P}<0.05 ;{ }^{\#}, \mathrm{P}<0.01 ;{ }^{\# \#}, \mathrm{P}<0.001$. AT-I, atractylenolide I. 
MyD88, and the ratio of $\mathrm{p}-\mathrm{IkB} / \mathrm{IkB}$ and $\mathrm{p}-\mathrm{p} 65 / \mathrm{p} 65$ were significantly suppressed in the AT-I-H and ABX + AT-I-H groups. These results indicate that the potential anti-tumor mechanism of AT-1 is related to the TLR4/MyD88/NF- $\mathrm{kB}$ signaling pathway and is mediated by intestinal microbes. In addition, compared with the AT-I group, the tumor volume and weight of the antibiotic + AT-I-H group were statistically significant, suggesting that the consumption of intestinal bacteria weakened the anti-tumor effect and intestinal diseases of AT-I in mice. The present study found that AT-I repressed the phosphorylation of $\mathrm{p} 65-\mathrm{NF}-\mathrm{\kappa B}$ as well as the downstream cytokines, IL-6 and IL-1 $\beta$, in dysbacteriosis mice. These results indicated that AT-I might affect the intestinal microbiome by regulating NF- $\mathrm{KB}$ signaling.

\section{Conclusions}

In conclusion, we report for the first time that AT-I can inhibit the dysbacteriosis by regulating the intestinal microbiome, probably through inhibiting TLR4/MyD88/ NF- $\mathrm{kB}$ signaling. The present study provides a basis for the application of AT-I as an alternative medication for treating gastrointestinal disorders.

\section{Acknowledgments}

Funding: This study was supported by the Shandong Traditional Chinese Medicine Science and Technology Development Plan Project (2019-0404).

\section{Footnote}

Reporting Checklist: The authors have completed the ARRIVE reporting checklist. Available at https://dx.doi. org/10.21037/atm-21-4656

Data Sharing Statement: Available at https://dx.doi. org/10.21037/atm-21-4656

Conflicts of Interest: All authors have completed the ICMJE uniform disclosure form (available at https://dx.doi. org/10.21037/atm-21-4656). The authors have no conflicts of interest to declare.

Ethical Statement: The authors are accountable for all aspects of the work in ensuring that questions related to the accuracy or integrity of any part of the work are appropriately investigated and resolved. Experiments were performed under a project license (No. 2020-039) granted by ethics board of Affiliated Hospital of Nanjing University of Traditional Chinese Medicine, in compliance with NIH guidelines for the care and use of animals.

Open Access Statement: This is an Open Access article distributed in accordance with the Creative Commons Attribution-NonCommercial-NoDerivs 4.0 International License (CC BY-NC-ND 4.0), which permits the noncommercial replication and distribution of the article with the strict proviso that no changes or edits are made and the original work is properly cited (including links to both the formal publication through the relevant DOI and the license). See: https://creativecommons.org/licenses/by-nc-nd/4.0/.

\section{References}

1. Khan MAW, Ologun G, Arora R, et al. Gut microbiome modulates response to cancer immunotherapy. Dig Dis Sci 2020;65:885-96.

2. Liu $X$, Wang $L$, Jing $N$, et al. Biostimulating gut microbiome with bilberry anthocyanin combo to enhance anti-PD-L1 efficiency against murine colon cancer. Microorganisms 2020;8:175.

3. Shui L, Yang X, Li J, et al. Gut microbiome as a potential factor for modulating resistance to cancer immunotherapy. Front Immunol 2020;10:2989.

4. Gu L, Xu Y, Xu W, et al. The exosome secretion inhibitor neticonazole suppresses intestinal dysbacteriosis-induced tumorigenesis of colorectal cancer. Invest New Drugs 2020;38:221-8.

5. Wan G, Xie M, Yu H, et al. Intestinal dysbacteriosis activates tumor-associated macrophages to promote epithelial-mesenchymal transition of colorectal cancer. Innate Immun 2018;24:480-9.

6. Xiao C, Xu C, He N, et al. Atractylenolide II prevents radiation damage via MAPKp38/Nrf2 signaling pathway. Biochem Pharmacol 2020;177:114007.

7. Li Y, Wang Y, Liu Z, et al. Atractylenolide I induces apoptosis and suppresses glycolysis by blocking the JAK2/ STAT3 signaling pathway in colorectal cancer cells. Front Pharmacol 2020;11:273.

8. Zhu C, Zhang L, Liu Z, et al. Atractylenolide III reduces NLRP3 inflammasome activation and Th1/Th2 imbalances in both in vitro and in vivo models of asthma. Clin Exp Pharmacol Physiol 2020;47:1360-7.

9. Wang K, Huang W, Sang X, et al. Atractylenolide I inhibits colorectal cancer cell proliferation by affecting 
metabolism and stemness via AKT/mTOR signaling. Phytomedicine 2020;68:153191.

10. Kim JH, Lee Y, Lee G, et al. Quantitative interrelation between Atractylenolide I, II, and III in Atractylodes japonica Koidzumi rhizomes, and evaluation of their oxidative transformation using a biomimetic kinetic model. ACS Omega 2018;3:14833-40.

11. Wang J, Nasser MI, Adlat S, et al. Atractylenolide II induces apoptosis of prostate cancer cells through regulation of AR and JAK2/STAT3 signaling pathways. Molecules 2018;23:3298.

12. Xu S, Qi X, Liu Y, et al. UPLC-MS/MS of Atractylenolide I, Atractylenolide II, Atractylenolide III, and Atractyloside A in rat plasma after oral administration of raw and wheat bran-processed atractylodis rhizoma. Molecules 2018;23:3234.

13. Ji Y, Kang Z, Kang N, et al. Atractylenolide III enhances the anti-neoplastic efficacy of docetaxel in gastric cancer cell by inhibiting fibroblast growth factor receptors 1 , -2, and -4 expression. J Environ Pathol Toxicol Oncol 2019;38:217-27.

14. Zhang R, Wang Z, Yu Q, et al. Atractylenolide II reverses the influence of lncRNA XIST/miR-30a-5p/ROR1 axis on chemo-resistance of colorectal cancer cells. J Cell Mol Med 2019;23:3151-65.

15. Patel T, Bhattacharya P, Das S. Gut microbiota: an indicator to gastrointestinal tract diseases. J Gastrointest Cancer 2016;47:232-8.

16. Shu Y'T, Kao KT, Weng CS. In vitro antibacterial and cytotoxic activities of plasma-modified polyethylene terephthalate nonwoven dressing with aqueous extract of Rhizome Atractylodes macrocephala. Mater Sci Eng C Mater Biol Appl 2017;77:606-12.

17. Xu Z, Cai Y, Fan G, et al. Application of Atractylodes Macrocephala Koidz Extract in Methicillin-Resistant Staphylococcus Aureus. Procedia Engineering 2017;174:410-5.

18. Wang R, Zhou G, Wang M, et al. The metabolism of polysaccharide from Atractylodes macrocephala Koidz and its effect on intestinal microflora. Evid Based Complement
Alternat Med 2014;2014:926381.

19. Ding S, Hu C, Fang J, et al. The protective role of probiotics against colorectal cancer. Oxid Med Cell Longev 2020;2020:8884583.

20. Peng M, Lee SH, Rahaman SO, et al. Dietary probiotic and metabolites improve intestinal homeostasis and prevent colorectal cancer. Food Funct 2020;11:10724-35.

21. Sivaprakasam S, Ristic B, Mudaliar N, et al. Hereditary hemochromatosis promotes colitis and colon cancer and causes bacterial dysbiosis in mice. Biochem J 2020;477:3867-83.

22. Guo H, Gibson SA, Ting JPY. Gut microbiota, NLR proteins, and intestinal homeostasis. J Exp Med 2020;217:e20181832.

23. Xu L, Zhang Y. Reviews on Antitumor Effects of Volatile Oil from Atractylodes Macrocephala Koidz. Journal of $\mathrm{Xi}^{\prime}$ an University of Arts and Science (Natural Science Edition) 2010;13:59-61.

24. Xu Z, Han L, Zhao Y. Studies on the Rare Anticancer Compounds of the Hydrolysate of the Saponins from Gynostemma pentaphfllum(Thunb)Makino. Asia-Pacific Traditional Medicine 2008;4:41-3.

25. Pan L, Song J. Study on inhibiting effects of atractylenolide I against melanoma in vitro and in vivo. Chinese Journal of Hospital Pharmacy 2015;35:682-5.

26. Wang CC, Lin SY, Cheng HC, et al. Pro-oxidant and cytotoxic activities of atractylenolide $\mathrm{I}$ in human promyeloleukemic HL-60 cells. Food Chem Toxicol 2006;44:1308-15.

27. Ye Y, Wang H, Chu JH, et al. Atractylenolide II induces G1 cell-cycle arrest and apoptosis in B16 melanoma cells. J Ethnopharmacol 2011;136:279-82.

28. Ding W, Zhou B, Zhai M, et al. Influence of Shenlinbaizhu Powder in enteric bacteria flora in mouse model with spleen-insufficiency syndrome. Journal of Beijing University of Traditional Chinese Medicine 2006;29:530-3.

(English Language Editor: A. Kassem)
Cite this article as: Liu P, Zhao G, Zhang L, Gong Y, Gu Y. Atractylenolide I inhibits antibiotic-induced dysbiosis of the intestinal microbiome. Ann Transl Med 2021;9(20):1539. doi: 10.21037/atm-21-4656 